\section{Cystic fibrosis two-step}

\section{By Chris Cain, Staff Writer}

A Canadian and a U.S. team have independently shown that restoring normal function to mutant CFTR requires correcting two distinct folding steps. ${ }^{1,2}$ The findings could provide a road map to guide the rational development of more effective therapies to treat cystic fibrosis.

$\mathrm{CF}$ is caused by genetic mutations in the cystic fibrosis transmembrane conductance regulator (CFTR), an ion channel that helps keep the lung epithelium hydrated and prevents mucous buildup that leads to airway obstruction and infection.

Last month, the FDA approved the first disease-modifying treatment for $\mathrm{CF}-$ Kalydeco ivacaftor from Vertex Pharmaceuticals Inc. Kalydeco is a small molecule CFTR potentiator that increases ion transport through the channel. The drug is indicated for CF patients with the G551D CFTR mutation, which decreases ion transport through the channel but does not impair its localization to the cell surface. This mutation occurs in about $4 \%$ of patients with CF.

About two-thirds of patients with CF, however, inherit two copies of a different mutation, the $\triangle F 508$ CFTR allele, which generates a misfolded version of the protein that is rapidly degraded and does not reach the surface of the cell in the first place. Because Kalydeco can only improve the function of CFTR that reaches the cell surface, distinct corrector compounds are needed to repair the folding defect caused by the $\Delta$ F508 mutation.

The only corrector in clinical development is VX-809, a small molecule from Vertex that is in Phase II testing in combination with Kalydeco in patients with $\Delta$ F508 CFTR. Data are expected this year. In vitro studies have suggested that Kalydeco and VX-809 can together restore up to about $25 \%$ of CFTR function, leaving open the question of whether further correction of CFTR folding could lead to additional improvement in $\Delta \mathrm{F} 508$ function. ${ }^{3}$

To answer this question, separate groups at The University of Texas Southwestern Medical Center and McGill University set out to use biophysical, computational and cell-based experiments to determine the exact mechanisms underlying $\Delta$ F508 CFTR misfolding.

The teams built upon work published by their labs in 2005 that suggested the $\Delta$ F508 mutation disrupts two distinct steps in CFTR maturation-the folding of CFTR's nucleotide binding domain 1 (NBD1), where F508 is located, and the additional domain-domain interactions between NBD1 and distinct structural regions within CFTR., ${ }^{4,5}$

"For the last 20 years or so, the dogma was that the predominant defect of $\Delta F 508$ was caused by the destabilization of the NBD1 domain," said Gergely Lukacs, professor of physiology at McGill. "When the crystal structure came out about seven years ago for $\Delta$ F508 NBD1, some doubt was cast on this theory."

Indeed, the structure suggested that the F508 residue may not play a central role in stabilizing NBD1 functionality, but rather that its location on an exposed surface of NBD1 may potentially affect NBD1's intramolecular interactions with other regions of the protein. ${ }^{6}$

Lukacs said that subsequent homology modeling of CFTR structure provided additional evidence that intramolecular interactions likely played a role in CFTR maturation. ${ }^{7,8}$

\section{Correcting by suppressing}

The unanswered question was whether it is possible to simultaneously correct both defects - the intramolecular destabilization and the compromised folding of the binding domain - and if so whether this would lead to a more substantial improvement in CFTR activity than stabilizing NBD1 alone.

Both teams independently sought to identify amino acid residues that interact at a structural level with the $\Delta$ F508 position and could therefore provide a path toward correcting the two defects and restoring CFTR folding.

The UT Southwestern team, led by professor of physiology Philip Thomas, performed a computational analysis of 493 CFTR-related gene sequences to identify amino acid residues whose variation was coupled to variation at the F508 position. This approach identified 16 residues that affected the folding of $\Delta \mathrm{F} 508 \mathrm{CFTR}$ in vitro.

Mapping the residues to the NBD1 crystal structure suggested a critical interaction between NBD1 and the fourth intracellular loop (ICL4) located in membrane spanning domain 2 (MSD2).

Lukacs' McGill group performed in vitro biophysical studies and reported that mutations that increased NBD1 stability in the $\Delta \mathrm{F} 508$ background did not lead to a proportional increase in CFTR maturation. This confirmed that an additional folding step must be altered in $\Delta$ F508 CFTR. Additional mutational analysis led them to pinpoint disruption of the NBD1-ICL4 interface as the likely second step.

Both teams tested suppressor mutations that either stabilized NBD1 folding or stabilized the NBD1-ICL4 interface and saw about a $20 \%$ increase in $\triangle F 508$ CFTR maturation. However, when they combined the two suppressor mutations, $\Delta \mathrm{F} 508$ CFTR maturation and function were restored to near wild-type levels in vitro.

Results from both studies were published in Cell.

"This work significantly moves the field forward-there was a huge amount of work in the papers. It really nails down the twostep hypotheses," said David Thomas, professor and chair of the Department of Biochemistry at McGill. "Because the previous crystal 
structures revealed very little difference between wild-type NBD1 and $\Delta$ F508 NBD1, they didn't lead us very far. Looking at the dynamic state of CFTR folding was key." Thomas was not involved in the studies.

"When we correct both steps together, instead of an additive effect, we see a synergistic effect," said Philip Thomas. "This suggests it would be desirable to correct both steps. The open question is whether there is a single small molecule out there, or one that could even exist, that is capable of doing that, because they are steps that are temporally isolated from each other."

\section{Searching for function}

Philip Thomas said the assays and mutations outlined in the two studies could provide a road map to identify how existing small molecule correctors function. "The use of mutations we identify will allow a better understanding of how they're acting, and that is one of the important features of this work. It provides an operations manual for determining the mechanism of action of extant correctors."

Almost all existing small molecule CFTR correctors have been identified through cell-based assays that screen for improvements in CFTR maturation and function but do not reveal detailed mechanisms of action.

"Our approach has been to do cell-based screens using chloride transport in cells as an endpoint, then to narrow them down using different criteria to select scaffolds for lead optimization," said Fred Van Goor, head of biology for Vertex's CF research program. "Phenotypic assays don't make any assumption on what folding state the compounds need to work on; they are just looking at the effect of compounds on CFTR cell surface localization and function."

VX-809 has been shown to be additive in vitro when combined with small molecule correctors identified by academics, but its molecular target and the CFTR folding step at which it functions have not been determined. ${ }^{3}$

David Thomas agreed that cell-based screens are the workhorse for discovery. "A lot of us are trying to come up with a rational way to look for synergy, but the brute force screening approach is being done too, and in some ways it is a lot easier to take that approach," he said.

In January 2011, David Thomas entered into a two-year collaboration with GlaxoSmithKline plc to screen for CFTR-correcting compounds. Also that year, his lab found a small molecule corrector of CFTR that directly bound to and stabilized mouse NBD1. ${ }^{9}$

Philip Thomas said the findings in the two studies could help researchers design more targeted screens. "For example, you could go to a cell-based assay and use the mutations we have identified here to screen for compounds that selectively correct step one and then look for compounds that could selectively fix step two. It informs a way to look for compounds that act at specific steps," he said.

Lukacs agreed and said his team's next steps include determining the mechanism of existing correctors and designing screening assays to identify correctors that act at each folding step.

"We can speculate that perhaps the drugs that have been developed so far predominantly target only one of these folding defects, because despite extensive efforts the maximum improvement seen in CFTR folding is around 15\%," said Lukacs.

Philip Thomas said he next plans to investigate the molecular pathology of additional CF mutations. He is also a cofounder of Reata
Pharmaceuticals Inc., which has licensed the in vitro screening assay that was used in his paper.

Chris Wigley, VP of research at Reata, would not disclose the company's plans for the screening platform. "The work described by these two independent labs suggests an elegant resolution to the so-called efficacy ceiling observed for corrector molecules that have been studied to date. Together, results of these studies support the potential of combination therapies that target the two defects in $\Delta \mathrm{F} 508$ CFTR maturation. It is attractive to speculate that with this information in hand, single agents can be designed that achieve similar functional correction."

Van Goor said that for patients with CF, full correction may not be necessary for clinical benefit. "What is clear from natural history studies of CF patients is that you don't need full amounts of CF function to have a difference in the clinical phenotype of disease progression. As little as $10 \%$ of normal function is present in individuals with much less severe CF disease."

Vertex is combining VX-809 with Kalydeco but does not currently plan to test corrector compound combinations. The company expects to begin a Phase II trial of VX-661, a second CFTR corrector, this quarter.

"Vertex is committed to several ongoing collaborations with the Cystic Fibrosis Foundation and their folding and structural consortium to understand the basic science underlying how VX-809 functions. We are continuing to work with the community to understand how these correctors work, which could lead to insights on how to improve correctors," he added.

The Cystic Fibrosis Foundation partially funded both studies, and the results are unpatented.

Cain, C. SciBX 5(8); doi:10.1038/scibx.2012.192

Published online Feb. 23, 2012

\section{REFERENCES}

1. Rabeh, W.M. et al. Cell; published online Jan. 20, 2012; doi:10.1016/j.cell.2011.11.024

Contact: Gergely L. Lukacs, McGill University, Montreal, Quebec, Canada

e-mail: gergely.lukacs@mcgill.ca

2. Mendoza, J.L. et al. Cell; published online Jan. 20, 2012; doi:10.1016/j.cell.2011.11.023

Contact: Philip J. Thomas, The University of Texas Southwestern Medical Center, Dallas, Texas e-mail: philip.thomas@utsouthwestern.edu

3. Van Goor, F. et al. Proc. Natl. Acad. Sci. USA 108, 18843-18848 (2011)

4. Du, K. et al. Nat. Struct. Mol. Biol. 12, 17-25 (2005)

5. Thibodeau, P.H. et al. Nat. Struct. Mol. Biol. 12, 10-16 (2005)

6. Lewis, H.A. et al. J. Biol. Chem. 280, 1346-1353 (2004)

7. Serohijos, A.W.R. et al. Proc. Natl. Acad. Sci. USA 105, 3256-3261 (2008)

8. Mornon, J.-P. et al. Cell. Mol. Life Sci. 65, 2594-2612 (2008)

9. Sampson, H.M. et al. Chem. Biol. 18, 231-242 (2011)

COMPANIES AND INSTITUTIONS MENTIONED

Cystic Fibrosis Foundation, Bethesda, Md.

GlaxoSmithKline plc (LSE:GSK; NYSE:GSK), London, U.K.

McGill University, Montreal, Quebec, Canada

Reata Pharmaceuticals Inc., Irving, Texas

The University of Texas Southwestern Medical Center,

Dallas, Texas

Vertex Pharmaceuticals Inc. (NASDAQ:VRTX), Cambridge, Mass. 